\title{
Millimetric Site Testing at Dome C: Results and Plans
}

\author{
Luca Valenziano
}

IASF - sezione di Bologna, via P. Gobetti 101, Bologna, Italy

Giorgio Dall'Oglio, Andrea Graziani, Lorenzo Martinis, Gabriella Pizzo, Lucia Sabbatini

Università di Roma TRE, via della Vasca Navale 84, Roma, Italy

\begin{abstract}
Results relative to three campaigns at Dome Concordia, aimed to measure the millimetric sky noise, are presented. The atmospheric noise during summer seems to be definitely lower with respect to that measured with the same instrumentation in other geographical locations. We illustrate the scientific results obtained.
\end{abstract}

\section{Introduction}

The high Antarctic Plateau is generally recognized as the best site on our Planet for millimetric and sub-millimetric observations. Italy and France are building a new permanent station at Dome C. It will be open for year-round operations in 2004-2005. The Dome C site quality in the millimetric range has been tested in summer since 1995. Recent data from atmospheric studies allow us to extend the analysis to wintertime conditions.

\section{Site testing observations}

Some interesting informations can be derived from meteorological data: wind speed, temperature, humidity. Wind speed at Dome C (median value below 2 $\mathrm{m} / \mathrm{s}$ ) is always lower than at South Pole (Valenziano \& Dall'Oglio 1999). This is mainly due to the relative location of the two sites: Dome $\mathrm{C}$ being on top of a dome, while South Pole is downhill, where katabatic regime is already present. Moreover, wind is almost absent at Dome $\mathrm{C}$ during the winter, being strongly correlated to the elevation of the Sun.

Temperature is strongly related to Sun elevation at Dome $\mathrm{C}$, being quite stable around $-60^{\circ} \mathrm{C}$ during the winter. Precipitable Water Vapor (PWV) is always very low at Dome C. Summertime values (Valenziano \& Dall'Oglio 1999) are distributed around $0.6 \mathrm{~mm}$. Relative humidity, measured with a set of capacitive sensors at three different location on an instrumented tower (Nardino et al. 2002), drops to very low values during the winter.

Planetary Boundary Layer observations, using SODAR and net radiation detectors (Argentini et al. 2002), show that the atmospheric turbulence is strongly related to the warming effect of the sun (Georgiadis et al. 2002). Net radiation (if positive is the the amount of energy available for turbulence genera- 

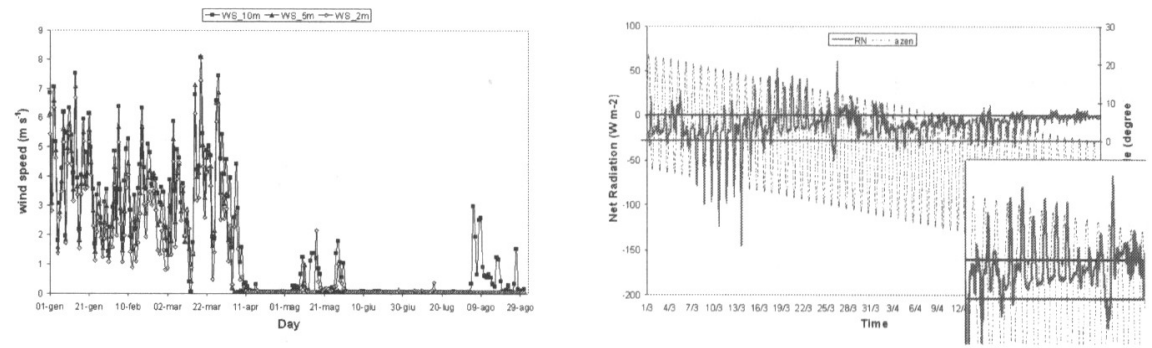

Figure 1. Left panel: Wind speed at Dome $\mathrm{C}$ in 2002. Note the abrupt change when the Sun is no longer above the horizon. Right panel: Net radiation measured at Dome C. Sun elevation is superimposed. Also in this case, values are dropping when the Sun is below the horizon. Note, in the box, the correlation between the net radiation and the Sun position, showing the presence of turbulence only during the central part of the day in summer. Data courtesy of M. Nardino, S. Argentini, T. Georgiadis (2002)

tion) is always very low and it is dropping below zero during the Antarctic night (see Figure 1). Moreover, the turbulent layer is confined close to the ground (lower than $300 \mathrm{~m}$ ) by the strong thermal inversion during the summer. This is also confirmed by sky noise data measured by the authors in summer 1998 .

\section{Conclusions}

While further test are in progress to definitively asses Dome C quality, present data strongly support the exceptional quality of the site in the millimetric range. The combination of very low PWV, wind speed, turbulence and temperature makes Dome $\mathrm{C}$ a very promising site for future, large aperture telescopes.

Acknowledgments. Authors are indebted to M. Nardino for kindly supplying Planetary Boundary Layer and meteorological data.

\section{References}

Argentini S., Mastrantonio, G., viola, A., Nardino, M., Petenko, I.V., Sempreviva, A.M. 2002 in SIF Conf. Proc., Italian Research on Antarctic Atmosphere, ed. M. Colacino, (Bologna: SIF) 80

Georgiadis, T., Argentini, S., Mastrantonio, G., Viola, A., Sozzi, R., Nardino, M. 2002, Nuovo Cimento, 25, 4

Nardino, M., Bonafè, U., Calzolari, F., Orsini, A., Argentini, S., Georgiadis, T. 2002 in SIF Conf. Proc., Italian Research on Antarctic Atmosphere, ed. M. Colacino, (Bologna: SIF) 80

Valenziano, L., Dall'Oglio, G. 1999, PASA, 16, 167 